\title{
PHARMACOTHERAPY OF PIOGLITAZONE AND ITS CURRENT STATUS IN INDIA
}

\author{
*Naveen Kumar T ${ }^{1}$, L Nagakrishna ${ }^{2}$, Amar Kumar $\mathbf{Y}^{3}$ \\ ${ }^{1}$ Associate professor, Dept of Pharmacology, Apollo Institute of Medical Science \& research, Jubilee Hills, Hyderabad, \\ A.P., India \\ ${ }^{2}$ Lecturer, Dept of Pharmacology, Malla reddy Institute of Medical Science, Hyderabad, A.P., India \\ ${ }^{3}$ Lecturer, Dept of Pharmacology, Kamineni Institutes of Medical Sciences, Narketpally, A.P., India
}

*Corresponding Author's Email ID; doctornaveen1@ rediffmail.com

\begin{abstract}
In the management of type-2 diabetis mellitus ie insulin resistance has become easier due to the discovery of Thiazolidinediones (also called Glitazones), other than Biguanides . However the adverse effects of Pioglitazone especially bladder cancer on long term use had made uncertain future regarding their safety. Purpose of this review article is to understand the pharmacology of Pioglitazone and its current status in India. Data was collected from Review articles, Standard pharmacology text books, Pharmacology journals and Electronic data base like Medline. They were analysed and relevant data was collected. There is no doubt at all regarding the therapeutic potential of the Glitazone group of drugs. Both Pioglitazone and Rosiglitazone have been hugely successful in controlling the HbAlc levels in a large number of patients with type 2 diabetes. Insulin resistance is more common in Asian indians and hence Glitazones have been very popular in India. However the correlation between Pioglitazone and bladder cancer is seen with duration of the therapy more than 24 months and cumulative dose of an average daily dose of Pioglitazone of about $40 \mathrm{mg} / \mathrm{day}$. In India, we generally do not use doses greater than $30 \mathrm{mg}$, which means, to achieve a cumulative dose we would take longer and if we use low dose $7.5 \mathrm{mg}$ Pioglitazone, whether Indians are equally, more, or less, prone to risk of bladder cancer than Europeans has to be further studied.

Keywords: Pioglitazone, Bladder cancer, PPARY agonist.
\end{abstract}

\section{INTRODUCTION}

Diabetis mellitus is a group of metabolic disorders characterized by chronic hyperglycemia associated with disturbances of carbohydrate, fat and protein metabolism resulting due to absolute or relative deficiency in insulin secretion, insulin action or both. Diabetis cause long term damage, dysfunction and failure of various organs especially the eyes, kidneys, nerves, heart and blood vessels. There is an increase in the prevalence of type -1 diabetis also, but main cause of diabetis epidemic is type-2 diabetis mellitus, which accounts for more than 90 percent of all diabetis cases. The majority of cases of diabetis fall into two broad etipathogenetic categories now called type 1 and type- 2 diabetis mellitus. The etiologic classification of diabetis mellitus currently recommended by WHO and the ADA in 1997.

\section{Diagnosis and Clinical Presentation}

Type 1 Diabetis Mellitus: Type -1 diabetes mellitus, results from insulin deficiency following destruction of the insulin-producing pancreatic beta cells and these patients depend on insulin for survival. On withdrawl of insulin they develop hyperglycemia, ketoacidosis and coma.It most commonly presents in childhood but one-fourth of cases are diagnosed in adults. The genetic factors, autoimmunity and environmental factors play a role in causation and precipitation of type-1diabetis mellitus. Symptoms are caused by hyperglycemia and include polyuria, polydipsia, and weight loss despite increased appetite initially. The absence or poor response of glucogon stimulated $\mathrm{C}$ peptide levels are diagnostic of type- 1 diabetis as these patients have low residual beta cell function.
Type 2 Diabetis Mellitus: T2 diabetis mellitus (T2DM) is the most common form of diabetes. The pathophysiological basis is a combination of impaired beta cell function, with marked increase in peripheral insulin resistance at receptor/post receptor levels and increased hepatic glucose output production. The risk of developing Type -2 diabetis mellitus increases with age, obesity, physical inactivity, and also shows strong familial tendency. C-peptide may be variable ranging from hyper to normoinsulinemic levels in a majority of the subjects.

\section{Monitoring Of Blood Glucose}

Blood Glucose Testing: The glucose con-centration is 10$15 \%$ higher in plasma or serum than in whole blood because structural components of blood cells are absent.

\section{Testing for Ketonuria/Ketonemia}

Most strips utilize a nitroprusside re-action that measures only acetone and acetoacetate. Although these tests do not detect $\beta$-hydroxybutyric acid, the semi quantitative estimation of the other ketone bodies is nonethe-less usually adequate for clinical assessment of ketonuria.

\section{Glycosylated Haemoglobin}

The major form of glycohaemoglobin (HbA1C) is abnormally elevated in diabetics. Glyco-haemoglobin generally reflects the state of glycemia over the preceding 8-12 weeks, thereby providing a method of assessing chronic diabetic control ${ }^{1}$.

\section{Management}


Diabetes mellitus is condition associated with number of complications including coronary heart disease, retinopathy, neuropathy etc. It is now clear that tight control of blood glucose significantly reduces the risk of complications of diabetes.

\section{Management of Type-2 Diabetis Mellitus}

Non-pharmacological Therapy: Non-pharmacological measures including diet, exercise and stress alleviation are important interventions for the management of diabetes.
Pharmacological therapy: Monotherapy( single drug ), combination of drugs (insulin resistant patients for type-2 DM) and Insulin (type- 1 DM ).

\section{PHARMACOLOGICAL THERAPY OF PIOGLITAZONE}

It indicated for the treatment of insulin resistant cases. Pioglitazone is probably the most potent and effective oral drug option among the five available groups, the sixth being insulin and the benefits of this 'insulin sensitiser' outweighs the risks.

The Mechanism of Action of Pioglitazone:

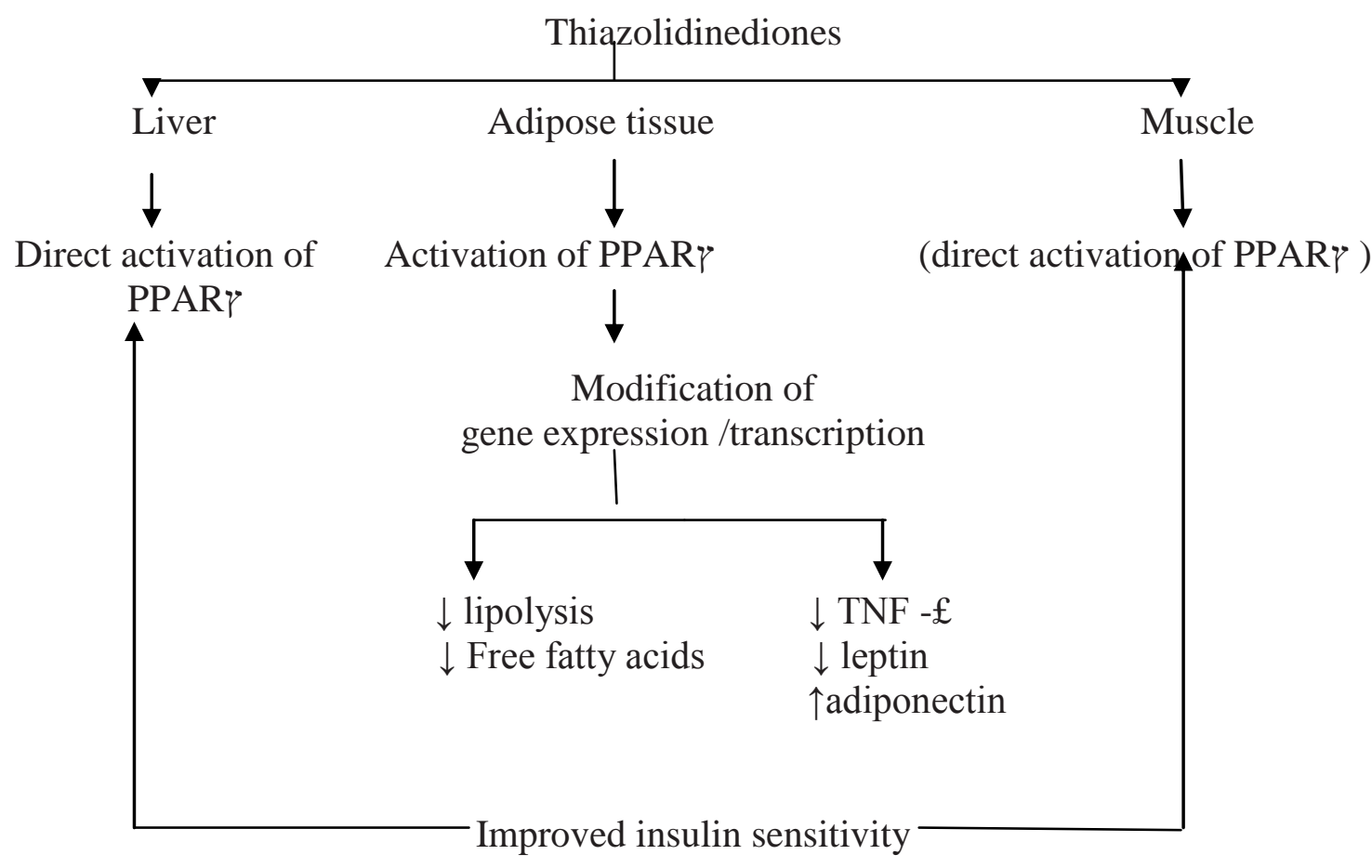

Thiazolidinediones are selective agonists for the nuclear perixosome proliferator -activated receptor $\gamma$ (PPAR $\gamma$ ) which is expressed mainly in fat cells, but also muscle and other cells. It enhances the transcription of several insulin responsive genes .Glitazones tend to reverse insulin resistance by enhancing GLUT 4 expression and translocation. Entry of glucose in to muscle and fat tissue is improved. Activation of genes regulating fatty acid metabolism and lipogenesis in adipose tissue contributes to the insulin sensitizing action. Lipolysis and plasma fatty acid levels are reduced. Adipocytes turn over and differentiation is accelerated by glitazones. Thus fatty tissue is major site of action ${ }^{2}$.

\section{THERAPEUTIC USES OF PIOGLITAZONE}

Pioglitazone improved glycaemic control results in lowering of circulating HBA1C (by $0.5 \%-1.2 \%$ ) without increasing circulating insulin levels in type-2 diabetis mellitus patients. About $25 \%$ patients may not respond (non-responders), probably due to low baseline insulin levels. It should be stopped if HBA1C reduction is less than $0.5 \%$ at 6 months. Pioglitazone is primarly used to supplement Sulfonylureas /Metformin and in case of insulin resistance. However, it is not likely to be effective when $\beta$ cell failure has set in, which may be the cause of loss of efficacy to a combination of Sulfonylureas /Metformin. Glitazones have beneficial effects beyond their blood glucose lowering properties ${ }^{3}$. Monotherapy with glitazones is not associated with hypoglycaemic episodes ${ }^{4}$.

Pioglitazone in addition lowers serum TG(26\% $\downarrow$ TG) and raises HDL( $14 \% \uparrow H D L)$ level without much change in LDL levels, propably because it acts on PPARY as well to induce expression of reverse cholesterol transporter and some apoproteins. One of the largest trials conducted on Pioglitazone, PROACTIVE showed that Pioglitazone improved glycemic control and additionally suggested a possible cardio-protective effect ${ }^{5}$. The PERISCOPE trial also showed that Pioglitazone was associated with improvement in $\mathrm{CV}$ risk factors and prevention of atherosclerosis progression compared to Glimepiride ${ }^{6}$. In the CHICAGO trial, Pioglitazone significantly slowed the progression of carotid intima-media thickness (CIMT) compared with Glimepiride ${ }^{7}$.

The Diabetes Prevention Programme (2005) has shown that glitazones have the potential to delay progression of pre-diabetics to overt type- 2 diabetis mellitus. They may help to conserve $\beta$ cell function in diabetis. Due to their remarkable efficacy in terms of glycemic control, they became popular and were widely used globally in spite of these side effects. 


\section{ADVERSE EFFECTS OF PIOGLITAZONE}

Pioglitazone is well tolerated however, both Pioglitazone and Rosiglitazone had their own spectrum of side effects like weight gain, decrease in haematocrit values, edema, heart failure, bladder cancer ,fractures and possible worsening of diabetic macular edema .

\section{Heart failure}

In heart failure Thiazolidinediones have different effect on cardiovascular out come.They cause fluid retention, edema ,exacerbate pre-existing heart failure but do not affect left ventricular function.Fluid retention respond to diuretics, perhaps Amiloride or Spironolactone in particular. 8,9

Indeed, in a detailed analysis of the patients in PROactive who developed serious heart failure subsequent all-cause mortality was proportionately lower with Pioglitazone (40/149 [26.8\%] versus 37/108 [34.3\%] with placebo; $\mathrm{p}=0.1338$ ). Significantly fewer Pioglitazone patients with serious heart failure went on to die, have a non-fatal MI or a stroke (34.9\% with Pioglitazone versus $47.2 \%$ with placebo; $\mathrm{p}=0.025)$. When considering the matter of heart failure in this context it is important to emphasise that the Pioglitazone group in general as well as those with serious heart failure, had significantly less death, MI and stroke ${ }^{10}$.

\section{Fractures}

Increased risk of fractures in females (0.8 fractures /100patients per year).Distal upper limb is more prone to fractures compared to distal lower limb.

\section{Myocardial infarction}

In patients taking Rosiglitazone increased incidence of myocardial infarction has led to drastic decline in the use of drug, and it being banned in several countries including India ${ }^{11}$.

\section{Hepatic dysfunction}

Few cases of hepatic dysfunction have been reported with Pioglitazone.Monitering of liver function is advised.It is contraindicated in liver disease and in congestive cardiac failure ${ }^{12}$. Pioglitazone is metabolised by both CYP2C8 and CYP3A4. Failure of oral contraception may occur during Pioglitazone therapy. Ketaconazole inhibits and Rifampin induce metabolism of Pioglitazone.

\section{Pregnanacy}

Pioglitazone should not be used during pregnancy because of teratogenecity.

\section{Bladder Cancer}

Diabetis itself increase risk of cancer including bladder. Pioglitazone in Preclinical studies had shown increased incidence of bladder cancer in male rats but initial experimental studies suggested that this might be a rat specific phenominan ${ }^{13}$. Indeed PPAR $\gamma$ is currently considered a potential target for both chemoprevention and cancer therapy based on some preclinical studies ${ }^{14}$ .Therefore, TZDs like Pioglitazone may increase, decrease or have a neutral effect on the risk of cancer or cancer progression in different species ${ }^{15}$.
Bladder cancer is estimated to occur in Indian men 4.8 per 100,000 persons , 20 per 100,000 persons in the US and this prevalence is believed to be higher in the US because of high dose use, and this is believed to be higher in people with diabetes.

A recent analysis published in Diabetis care, reports on data on bladder cancer associated with antidiabetic drug use retrieved from the US FDA Adverse Event Reporting System (AERS) ${ }^{16}$.

The Kaiser Permanente study in California, USA22 included 1,93,099 patients aged $\geq 40$ years of age at study entry. The median duration of therapy among Pioglitazone-treated patients was 2 years (range 0.2-8.5 years). The results showed that after adjusting for age, sex, use of tobacco products, use of other categories of diabetes medications, and other risk factors, the risk of bladder cancer increased with increasing dose and duration of Pioglitazone use. Patients who were on Pioglitazone therapy for longer than 24 months, were found to have a $40 \%$ increased risk of bladder cancer ${ }^{17}$.

The US FDA has also asked to revise the patient medication guide for Pioglitazone including information regarding the increased risk of bladder cancer. FDA has further recommended that healthcare professionals should not use Pioglitazone in patients with active bladder cancer and to use it with caution in patients with a prior history of bladder cancer. Finally, the FDA has stated that additional information should be provided to patients stating that they should report to a physician if there are any symptoms of bladder cancer such as blood or red color in urine, urgent need to urinate or pain while urinating, pain in back or lower abdomen.

A Canadian team of researchers decided to investigate if Pioglitazone was linked to a higher risk of bladder cancer in people with type 2 diabetes and after obtaining data from the General Practice Research Database (GPRD), they evaluated 115,727 patients who initiated diabetes therapy from 1988 to 2009. The GPRD contains anonymous patient records from over 600 UK GPs. The team identified cases of bladder cancer and matched them to up to 20 healthy control patients.

The results revealed that from 376 cases that were matched against 6,699 controls, 470 patients were diagnosed with bladder cancer during the average 4.6 years of follow-up, which translates to a rate of 89 per 100,000 person years, whilst the rate of bladder cancer in the general UK population aged 65 years and above is 73 per 100,000 person years.

Patients who had taken Pioglitazone at any time were found to have a $83 \%$ higher risk of bladder cancer, which translates into 74 per 100,000 person years. The figures increased to 88 per 100,000 person years for those who had taken the drug for two years or longer and increased even further, to 137 per 100,000 person years those who had taken $28,000 \mathrm{mg}$ or more.

Researchers cross-checked the results in several further analyses, but the results remained consistent, and "provide evidence that Pioglitazone is associated with an increased risk of bladder cancer, whereas no increased risk was observed with the Thiazolidinedione Rosiglitazone." 
They believe that these links may have been underestimated during earlier observational studies and comment that doctors, patients and regulatory agencies "should be aware of this association when assessing the overall risks and benefits of this therapy."

Safety and efficacy of low dose Pioglitazone $(7.5 \mathrm{mg} /$ day) vs standard dose $(15 \mathrm{mg} /$ day $)$ in Japanese women with type-2 diabetis mellitus suggested low dose Pioglitazone could be a good choice for type- 2 diabetis mellitus in Japanese women.The incidence of peripheral edema in group A (with low dose) was low when compared with group -B .In addition \% change of body weight in group A was significantly less than group ${ }^{18}$.

In contrast, the European Medical Agency alert concludes that the benefit/risk balance remains positive in favour of Pioglitazone in a limited population of patients with type 2 diabetes and that the small increased risk of bladder cancer could be reduced by appropriate patient selection. However, this agency also recognizes the need for periodic review of the efficacy and safety of the individual patient's treatment.

\section{CURRENT STATUS OF PIOGLITAZONE IN INDIA:}

With the recent ban of the popular drug Pioglitazone by the health ministry in 2013 , there are number of questions about the reasons for their ban. One reason is the correlation between Pioglitazone and bladder cancer is seen with duration of the therapy more than 24 months and a cumulative dose of more than $28,000 \mathrm{mg}$ which corresponds to an average daily dose of pioglitazone of about $40 \mathrm{mg} / \mathrm{day}$. The doses used in US and Europe are 30-45 mg/day. In India, we generally do not use doses greater than $30 \mathrm{mg}$, which means, to achieve a cumulative dose of $28,000 \mathrm{mg}$, we would take longer and if we use low dose $(7.5 \mathrm{mg})$ Pioglitazone, it would take about 10 years. Indeed, low dose Pioglitazone has of late become very popular in India due to its efficacy and lower frequency of side effects like weight gain and fluid retention. However, we do not know whether Indians are equally, more, or less, prone to risk of bladder cancer than Europeans.

As per the research reports from various diabetis research centres in India, eight cases of bladder cancer in patients with type- 2 diabetes on Pioglitazone have been reported. Three of the patients were from Chennai, two from Salem and one each from Belgaum, Hyderabad and Mumbai. Seven of the eight patients were males. The patients ranged in age from 43 years to 76 years. They all had type 2 diabetes and were on Pioglitazone for periods ranging from two to nine years.

"The mean dose of Pioglitazone ranged from $15 \mathrm{mg}$ to 30 mg per day. While seven patients had transitional cell (urothelial) carcinoma on biopsy, the nature of malignancy was not clear in the eighth patient. Seven patients are presently on follow-up with urologists and oncologists, whereas the eighth patient developed metastases to the liver and lungs and died in November 2011 after several cycles of chemotherapy and two surgeries. The single female patient developed bladder cancer seven years ago while on $30 \mathrm{mg}$ of Pioglitazone."
"She underwent surgery for the same in the US and was declared free of malignancy. Since the link of Pioglitazone with bladder cancer was not known at that time, she was continued on Pioglitazone even after the surgery. She developed recurrence of haematuria and pain four months later and had to undergo repeat surgery and chemotherapy."

"This is, to the best of our knowledge, the first report on bladder cancer among Pioglitazone users in India and one of the first in a female patient. Although one cannot draw any conclusions of causality from these eight cases, the development of this relatively uncommon malignancy in eight patients who were on this particular drug does raise concern about the long-term safety of Pioglitazone. Clinicians should therefore exercise extreme caution in the use of this drug. Patients should be made aware of this potential side-effect of Pioglitazone and should be allowed to make informed choices regarding its use,"

The US Food and Drug Administration warned in June 2011 that taking Pioglitazone for at least a year increased the risk of bladder cancer by at least $40 \%$, and ordered the drug's manufacturer to note the risk on the drug's label. The drug is banned in some places like France and Germany.

Cancer as adverse effects of many drugs is difficult to prove for several reasons. Cancers have multifocal etiologies like genetic interactions, exposure to chemicals, environmental factors, diagnosed after considerable time has passed. All thse factors make it impossible to point out a certain drug as a potential carcinogenic drug.

For the possible risk of bladder cancer with Pioglitazone physicians have to be careful and explain to patients regarding risk of bladder cancer. When prescribing Pioglitazone it should be started with low dose of $7.5 \mathrm{mg} /$ day and should not exceed maximum dose of 15 $\mathrm{mg} /$ day (toxic doses for bladder cancer in India as reported between $15 \mathrm{mg}$ to 30mgper day). If blood sugar is not controlled with maximum dose of $15 \mathrm{mg} /$ day add on therapy with other 'insulin sensitiser' drug can be done. Other risk factors are whether there is a history of any cancer in the family or patient he has cancer should be considered and drug should be withdrawn.

Councel patients to report any signs or symptoms of blood in the urine, urinary urgency, pain on urination, or back or abdominal pain, as these may be due to bladder cancer. Prescribers are advised not to use these medications in patients with current or history of bladder cancer or in patients with uninvestigated macroscopic haematuria.They should also consider the age of patient and review the treatment after 3 to 6 months and regularly after wards.

\section{SUMMARY AND CONCLUSION}

In India we require more studies for risk of bladder cancer with Pioglitazone, till that time, we may continue to use low dose of $7.5 \mathrm{mg}$ and dose should not exceed $15 \mathrm{mg} /$ day as it is a very good 'insulin sensitiser' and benefit ratio outweighs risk ratio as glucose lowering agent.The patient should also be informed about adverse effects of the drug with careful monitoring and follow up. At present there is on-going discussion by the ministry of health in India on the Pioglitazone ban taking views from different 
diabetoligists and general practioners all over India regarding the ban of the drug, and in the future the ban

\section{REFERENCES}

1.Stephen Mcphee J,Maxine Papadakis A, Michael Rabow W.Diabetis Mellitus And Hypoglycemia.In :Current Medical Diagnosis And Treatment,51 ${ }^{\text {st }}$ ed.New Delhi:Mc Graw Hill Medical ;2012.p.1167.

2.Tripathi KD. Insulin ,Oral Hypoglycaemic Drugs and Glucagon.In:Tripathi KD.Essentials of Medical Pharmacology, $7^{\text {th }}$ ed .New Delhi :Jaypee Brothers Medical Publishers (p) Ltd; p. 277.

3. Clifford Bailey J, Andrew Krentz J. Ora Antidiabetic Agents. In :Textbook of Diabetes. $4^{\text {th }}$ Edition. Wiley Blackwell Publication; 2010;452-77.

4. Tripathi KD. Insulin ,Oral Hypoglycaemic Drugs and Glucagon.In:Tripathi KD.Essentials of Medical Pharmacology, $7^{\text {th }}$ ed .New Delhi :Jaypee Brothers Medical Publishers (p) Ltd; p. 277.

5. Dormandy JA, Charbonnel B, Eckland DJ. Secondary prevention of macrovascular events in patients with type 2 diabetes: a randomized trial of pioglitazone, the proactive Study . Lancet 2005;366:1279-89.

6.Nissen SE, Nicholls SJ, Wolski K. Comparison of pioglitazone vs. glimepiride on progression of coronary atherosclerosis in patients with type 2 diabetes. The periscope randomized controlled trial. JAMA 2008;299:1561-73.

7. Mazzone T, Meyer PM, Feinstein SB. Effect of pioglitazone compared with glimepiride on carotid intima-media thickness in type 2 diabetes. A randomized trial. JAMA 2006 ; 296:2572-81.

8. Nesto RW, Bell D, Bonow RO, Fonseca V, Grundy SM, Horton ES, et al. Thiazolidinedione use, fluid retention, and congestive heart failure: a consensus statement from the American Heart Association and American Diabetes Association. Circulation 2003;108:2941-8.

9. Singh S, Loke YK, Furberge D.Thiazolinediones and heart failure -ateleo-analysis . Diabetes care 2007;30:2148-53.

10. Erdmann E, Dormandy JA, Charbonnel B. The effect of pioglitazone on recurrent myocardial infarction in 2,445 patients may be revoked.

with type 2 diabetes and previous myocardial infarction: results from the proactive (proactive 05) Study. J Am Coll Cardinol 2007;49:1772-80.

11. Nissen SE, Wolski K. Effect of rosiglitazone on the risk of myocardial infarction and death from cardiovascular causes. New England Journal Of Medicine 2007;356:2457-71.

12. Alvin Powers C, David Alessio D.Endocrine Pancreas and Pharmacotherapy of Diabetes Mellitus and Hypoglycemia.In:Laurence Bruton L,Goodman and Gilmans The Pharmacological Basis of Therapeutics, $12^{\text {th }}$ ed.New Delhi :Mc Graw Hill Medical ; 2011.p.1261.

13. Suzuki S, Arnold LL, Pennington KL, et al. Effects of pioglitazone, a peroxisome proliferator-activated receptor gamma agonist, on the urine and urothelium of the rat. Toxicology Science 2010;113:349-57.

14. Panigrahy D, Huang S, Kieran MW, Kaipainen A. PPAR $\gamma$ as a therapeutic target for tumour angiogenesis and metastasis. Cancer Bol Ther 2005;4:687- 93.

15. Clay CE, Namen AM, Atsumi G . Magnitude of peroxisome proliferator-activated receptor gamma activation is associated with important and seemingly opposite biological responses in breast cancer cells. J Investig Med 2001;49:413- 20.

16. Piccinni C, Motola D, Marchesini G, Poluzzi E. Assessing the Association of Pioglitazone Use and Bladder Cancer Through Drug Adverse Event Reporting. Diabetes Care 2011;34:136971

17. Lewis J, Quesenberry Jr C, Ferrara A, Vaughn D, Peng T. Risk of bladder cancer among diabetic patients treated with pioglitazone.Diabetes Care 2011;34:91-92.

18. Majima T, Komatsu Y,Doi K, Shigemoto M, Takagic C, Fukao A etal. Safety and efficacy of low dose Pioglitazone $(7.5 \mathrm{mg} /$ day $)$ vs.Standard dose ( $15 \mathrm{mg} /$ day) in Japanese women with type- 2 diabetis mellitus.2006 june ; 53(3):325-30. 